\title{
Norwegian Language
}

National Cancer Institute

\section{Source}

National Cancer Institute. Norwegian Language. NCI Thesaurus. Code C154059.

A North Germanic language that is the official language of Norway. 